\title{
Detection of ultrasonic plate waves using ceramic strip transducers
}

\author{
Leander Claes, Fabian Bause, Jens Rautenberg, Bernd Henning \\ Measurement Engineering Group, University of Paderborn, Warburger Str. 100, 33098 Paderborn, \\ Germany, \\ claes@emt.uni-paderborn.de
}

\begin{abstract}
:
By examining the propagation characteristics of acoustic waves through a specimen its material properties can be determined. One method to realize such a measurement is the excitation and detection of ultrasonic waves in plate-like waveguide structures. Therefore, a transducer with a small active area is required to detect guided waves with a small wavelength. However, the output voltage of piezoelectric transducers is approximately proportional to its active area. To circumvent this problem transducers with an active area that ideally resembles a line are used [1]. If these detectors are aligned with the wave front of the propagating waveguide modes, the lower bound of the detectable wavelength can be minimized due to the transducer's minimum extent in propagation direction. As an alternative to transducers based on piezoelectric polymer films, a ceramic strip transducer is presented. In order to verify the functionality of the transducer selected measured results and their evaluation are shown.
\end{abstract}

Key words: piezoceramics, strip transducers, plate waveguide, dispersion diagram

\section{Introduction}

As a means of non-destructive testing the influence of a material's properties on acoustic waves can be examined. A method to excite ultrasonic waves is by utilizing the photoacoustic effect. Pulsed laser radiation is used to heat up the material on the surface of a plate waveguide. The resulting thermal expansion creates strains in the material, which generate a broadband acoustic wave. Focusing the laser radiation can minimize the excitation area of ultrasound. The detection of the acoustic wave with high temporal and spatial resolution is more challenging. On the one hand, the extent of the transducer in the propagation direction poses as a lower bound on the detectable wavelength comparably to the Nyquist criterion. On the other hand, a small active area of the piezoelectric transducers tends to result in smaller amplitudes of the output signals. To circumvent these limitations transducers with an active area that ideally resembles a line are used. If these linetransducers are aligned with the phase front of the acoustic wave to be detected, the limitation of the detectable wavelength can be minimized. Typically, a piezoelectric polymer film, which is pressed on the surface of the specimen by a metal blade, is used to realize such transducers
[1]. In this paper, an alternative realization using a piezoelectric ceramic is presented. Limits given by the finite expansion of the transducer's active area in propagation direction of the acoustic wave are discussed.

\section{Realization of the ceramic strip transducer}

For the construction of a strip transducer ceramics of the type PIC255 (PI Ceramics, Germany) are used. The piezoceramic's dimensions are $25 \mathrm{~mm}$ by $1 \mathrm{~mm}$, with a thickness of $0.5 \mathrm{~mm}$. To dampen the resonances of the ceramic strip transducer it is embedded in a trapezoid block of polyurethane mixed with tungsten carbide [2] (see Fig. 1). The front of the ceramic protrudes about $0.1 \mathrm{~mm}$ from the damping body.

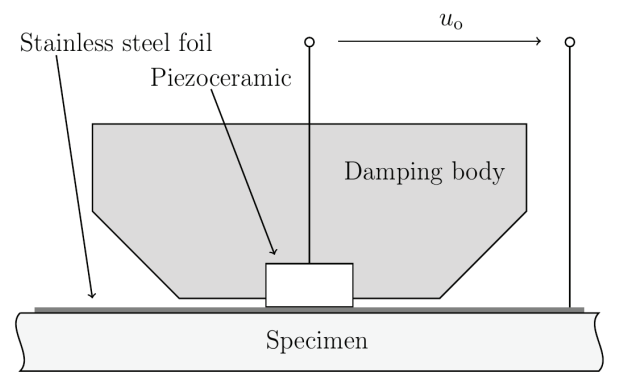

Fig. 1. Cross-section of the ceramic strip transducer. 
The trapezoid shape is used to prevent waves that are reflected from the sides of the block from being propagated back to the ceramic. Before casting the damping body, a wire is connected to the back electrode of the piezoceramic. The electric contact to the piezoceramic's front electrode is realized by placing a thin stainless steel foil with $5 \mu \mathrm{m}$ in thickness between the specimen and the transducer. A small amount of couplant ensures contact of the materials. If the specimen is metallic, connecting the specimen itself can alternatively make the electric contact. To yield reproducible results of the measurement the pressure applied to the transducer to make contact to the specimen is kept constant.

\section{Impedance of the piezoceramics}

Fig. 2 shows the absolute value of the piezoceramic's electrical impedance before and after the casting of the damping body. The undamped piezoceramic shows resonanceantiresonance-pairs at $60 \mathrm{kHz}$, at $1.5 \mathrm{MHz}$ and at $2.6 \mathrm{MHz}$, which correspond with the resonances of the ceramic in the three spatial directions. The first resonance shows at about $60 \mathrm{kHz}$ and can be assigned to the oscillation of the ceramic in longitudinal direction $(25 \mathrm{~mm})$. The resonance-antiresonance-pairs at $1.5 \mathrm{MHz}$ and $2.6 \mathrm{MHz}$ correspond to the oscillation in width direction $(1 \mathrm{~mm})$ and thickness direction $(0.5 \mathrm{~mm})$, respectively. For usage as a broadband receiving transducer, these resonances have to be damped. After embedding the ceramic in polyurethane mixed with tungsten carbide, only the oscillation in width direction remains visible in the ceramics frequency-dependent impedance plot.

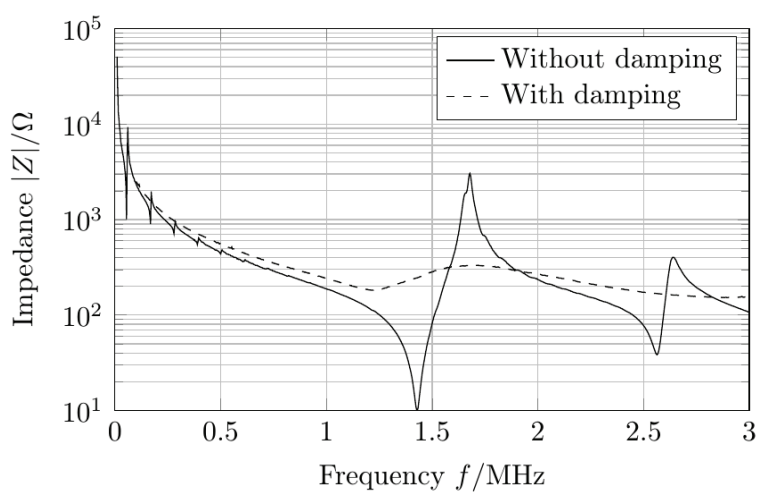

Fig. 2. Absolute value of the ceramic's electrical impedance before and after embedding in the damping body.

\section{Restriction of the detectable wavelength by the finite expansion of the transducer}

Compared to transducers based on blades and piezoelectric films the ceramic strip transducer's extension in propagation direction of the wave to be detected is larger and limits the detectable wavelength. It is assumed that waves with a wavelength $\lambda$ that is more than twice the transducer's width $w_{\text {transd }}$ are recorded unattenuated:

$$
\lambda \geq 2 w_{\text {transd }}
$$

This can alternatively be expressed as an upper bound for the detectable angular wave number $k$ :

$$
k \leq \frac{\pi}{w_{\text {transd }}}
$$

Given the width of the ceramic $(1 \mathrm{~mm})$, the cutoff angular wavenumber is $3142 \mathrm{rad} / \mathrm{m}$. However, no expression for the limit of the spectral bandwidth of the transducer can be given, as the relation between wave number and frequency is non-linear and dependent on the mode of the acoustic wave. It can be obtained by numerically solving the RayleighLamb frequency equation [3].

\section{Experimental setup}

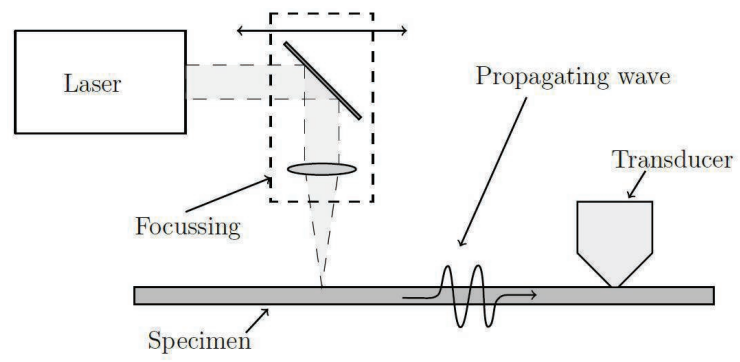

Fig. 3. Experimental setup for the excitation and detection of ultrasonic waves in plates.

To examine the detection properties of the ceramic strip transducer the experimental setup shown in Fig. 3 is used. The laser radiation

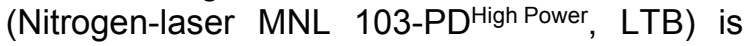
focused on a line on the specimen's surface using a cylindrical lens. The excitation on a line creates acoustic waves with straight phase fronts. This way, the distortion of the signal, which would be caused by examining circular waves using a linear detector, can be minimized. The optical elements (the cylindrical lens and a front surface mirror) are mounted on a linear actuator (T-LSM, Zaber Technologies Inc.) to adjust the distance between the line of excitation and detection of the acoustic wave. This is required to pick up spatially resolved measurement data and can be used to observe the changes in the ultrasonic impulse's shape as it travels through the specimen. The transducer is placed on the surface of the 
specimen with the ceramic strip aligned in parallel to the line of excitation. An amplifier with a bandwidth from $5 \mathrm{kHz}$ to $1 \mathrm{MHz}$ and a gain of 500 is used to increase the voltage of the measured signals.

\section{Measurement results}

Fig. 4 shows an amplified output signal of the ceramic strip transducer. An ultrasonic pulse that was excited by utilizing the photoacoustic effect is recorded. The distance between the location of the excitation and the transducer is $50 \mathrm{~mm}$. The specimen is a $1.53 \mathrm{~mm}$ thick copper plate. Before the dominant signal group, which arrives at the transducer at about $t=$ $15 \mu \mathrm{s}$ an oscillation with a smaller peak voltage is visible (at $t=10 \mu \mathrm{s}$ ). As this part of the ultrasonic pulse arrives at the transducer sooner and has a visibly higher frequency one can assume that it is transmitted in a different mode (SO) than the dominant signal group (AO).

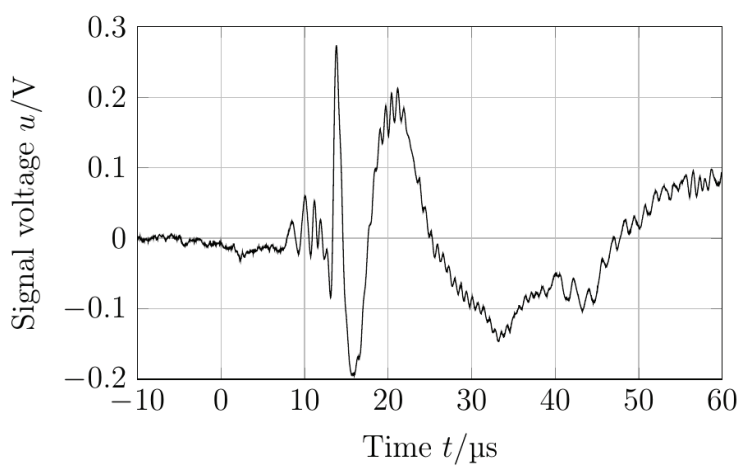

Fig. 4. Time signal of an ultrasonic pulse generated utilizing the photoacoustic effect recorded using the ceramic stripe transducer.

To further investigate the properties of the waveguide, spatially and temporally resolved measurements of the displacements of the specimen's surface are required. For this kind of measurement, the distance between excitation and detection of the ultrasonic pulse is increased in equidistant steps. A time signal is recorded for each step and the resulting data is arranged in a two-dimensional matrix (see Fig. 5). It is assumed that the signal voltage is proportional to the displacement of the waveguide's surface on location of measurement. The columns in this plot are interpreted as the displacement of the surface for a specific time, while the lines are the time signals. If one examines the time signals for a specific shift $\Delta x$, there are two distinguishable signal groups, which are recorded by the transducer at different times t. For $\Delta x=40 \mathrm{~mm}$ the first group arrives at about $30 \mu \mathrm{s}$, while the second, stronger signal group arrives at $45 \mu \mathrm{s}$. Because this difference between their time of flights increases with the shift of the excitation, one can conclude that the signals are transmitted at different group velocities. This suggests that the signals are transmitted through different propagation modes. Given the asymmetric nature of the excitation of the wave, and the fact that the slower mode has a higher amplitude, one can conclude that the weaker, faster impulse is transmitted via the S0-mode, and the stronger but slower impulse is transmitted via the A0-mode. In addition, it is to be noted that the phase of the faster signal group undergoes no visible change. This shows that the group and phase velocity of the fast propagation mode hardly differ. The phase of the slow signal group varies notably, which indicates a bigger difference between phase and group velocity and thereby a dispersive propagation of the impulse.

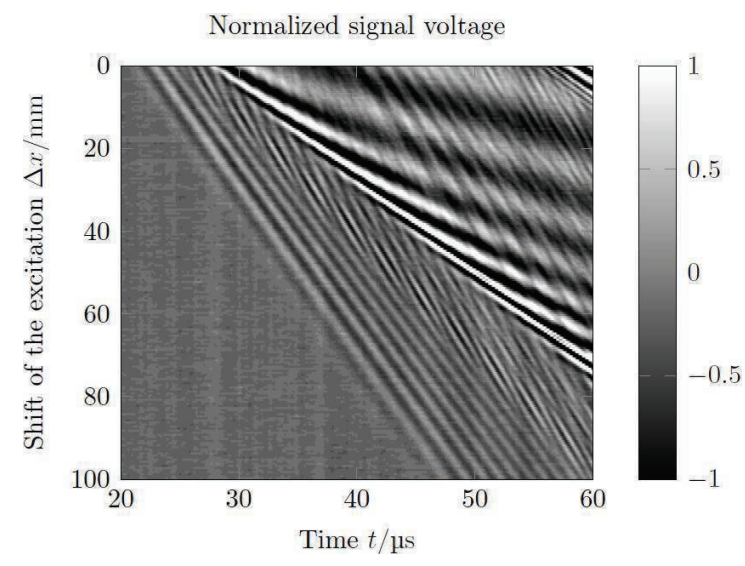

Fig. 5. Matrix of spatially and temporally resolved, measured data (signal voltage), recorded using the piezoceramic strip transducer.

Performing a two-dimensional Fourier transform on the matrix (Fig. 5) yields a depiction in the wavenumber/frequency regime (Fig. 6). This dispersion map shows ridges where curves are expected in the angular wavenumber's dispersion plot [4]. To increase the visibility of the ridges in the dispersion map, the depiction is displayed logarithmically. Due to this, the aforementioned damping to waves with $k>3142 \mathrm{rad} / \mathrm{m}$ is not directly visible. To further improve the visibilty of the ridges in the dispersion map, several measures are taken. A window function is applied to the time signals to eliminate the influence of signal groups that are caused by reflections of the acoustic wave on the specimen's edges. The shape of the window function has to be adapted depenting on the distance between excitation and detection of the acoustic wave, as the ultrasonic impulse reaches the transducer at different times accordingly. Further, a two-dimensional low-pass filter can be used to remove noise 
from the depiction. If there are interferences from other sources (amplifier noise, net frequency, switching power supplys, ect.) that are independent of the distance between excitation and transducer, they manifest as vertical lines in the frequency-wavenumber-plot. To remove them, the mean of each column is calculated and substracted from the respective column.

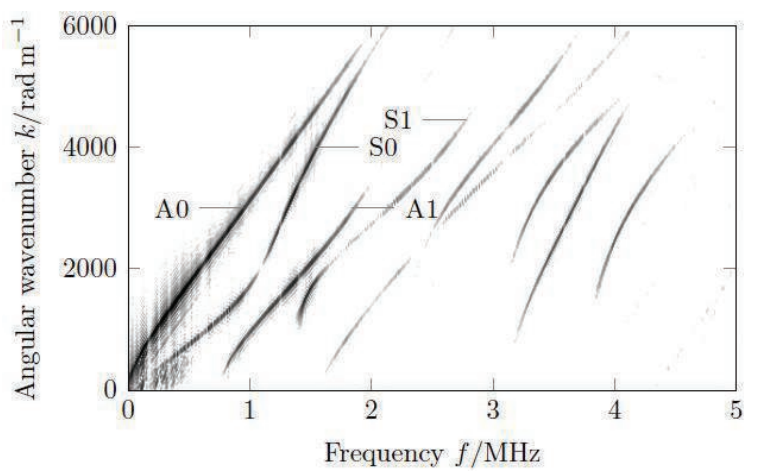

Fig. 6. Dispersion map of a copper plate (thickness $1.53 \mathrm{~mm}$ ). Generated by applying the 2D-FFT to spatially and temporally resolved data.

Fig. 6 shows a dispersion map that is generated by applying the 2D-FFT to the measurement data in Fig. 5. The visibility of the ridges is enhanced by the aforementioned measures. Ridges corresponding with nine propagation modes are visible. The robustness of this method is further shown by the fact that the last detected mode shows at about $4 \mathrm{MHz}$, which ist far beyond the cut-off frequency of the amplifier used.

Further analysis of the recorded data requires ridge-detection. A recursive algorithm is used to extract the positions of the ridges and convert the dispersion map to sections of the dispersion curves of the waveguide. These are used to calculate the phase velocity $V_{\mathrm{ph}}$ of the respective modes:

$$
v_{\mathrm{ph}}=\frac{\omega}{k}=\frac{2 \pi f}{k}
$$

The dispersion diagram of the phase velocity is compared to simulation results, which are generated by solving Lamb's equations [3] using an isotropic elastic material model (see Fig. 7). In an inverse procedure, the model parameters used in the simulation are adapted to match the calculated results and the simulation. The resulting model parameters are used as an approximation of the waveguides material properties. For the examined copper specimen the adapted model parameters are as follows:

- Longitudinal sound velocity $c_{1}=4875 \mathrm{~m} / \mathrm{s}$
- Transversal sound velocity $c_{\mathrm{t}}=2342 \mathrm{~m} / \mathrm{s}$

- Density $\rho=8900 \mathrm{~kg} / \mathrm{m}^{3}$

- Thickness $t=1.53 \mathrm{~mm}$

With these parameters, the following approximation for the material properties are calculated [5]:

- Poisson's ratio $v=0.35$

- Shear modulus $G=48.8 \mathrm{GPa}$

- Young's modulus $E=131 \mathrm{GPa}$

While Poisson's ratio and shear modulus of the specimen are mostly concurring with literature, Young's modulus deviates upwards [6] [7].

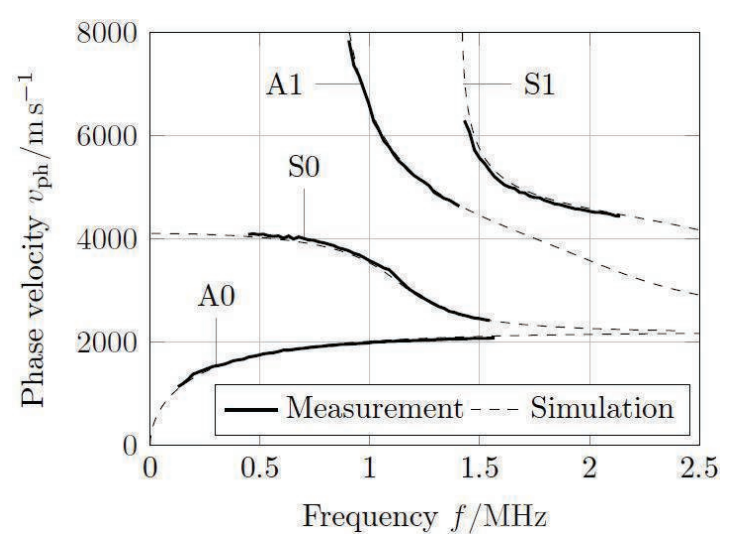

Fig. 7. Comparison of the phase velocity, calculated from the measurement results (2D-FFT, ridge-detection) and the result of simulation (Lamb's equations [3]) using the determined model parameters.

\section{Conclusion and outlook}

As shown by the measurement results, piezoceramic strip transducers can be used to detect ultrasonic plate waves. In the presented exemplary measurement, the utilized amplifier limits the bandwidth of the detected waves. An upper limit of the detectable angular wavenumber is imposed by the width of the piezoceramic strip. The theoretical consideration yields that angular wave numbers above $3142 \mathrm{rad} / \mathrm{m}$ are recorded attenuated. Measurements show, however, that wave numbers up to $6000 \mathrm{rad} / \mathrm{m}$ can be recorded (Fig. 6). The acquired temporally and spatially resolved data is processed to yield a dispersion map of the specimen via a two-dimensional Fourier transform. Comparing the result of the measurement to simulation results yields approximations for the specimen's material properties. The piezo ceramic strip transducer can accordingly be used to detect plate wave for the determination of the waveguide's dispersive properties. 
Further studies should include a comparison of the ceramic strip transducer and a transducer that utilizes a piezoelectric polymer. A monolithic design of the sensor should be developed in order to avoid the use of stainless steel foil for contacting. The length of the ceramic strip could be reduced to lessen the signal distortion that is caused by plate waves with curved phase fronts. In the given example, a metallic specimen is used. It is to be determined, if the transducer's sensitivity suffices to detect waves in materials with higher damping, such as polymers. In further studies, the effects of a paint or fluid layer on the waveguide surface can be analysed. The measurement set-up may also be used to investigate the directional dependence of the wave propagation in a waveguide, as it occurs in anisotropic or inhomogeneous materials, such as fibre-reinforced polymers.

\section{Acknowledgements}

The authors would like to thank the German Research Foundation (DFG) for financial support of the research project HE 2897/3-1 (determination of acoustic material properties).

\section{References}

[1] H. Coufal, R. Grygier, P. Hess, A. Neubrand, Broadband detection of laser-excited surface acoustic waves by a novel transducer employing ferroelectric polymers, The Journal of the Acoustical Society of America 92, 2980-2983 (1992); doi: 10.1121/1.404363

[2] F. Bause, J. Rautenberg, B. Henning, Design, modeling and identification of an ultrasonic composite transducer for target impedance independent short pulse generation, 16. Internationaler Kongress für Sensoren und Messtechnik (Sensor 2013), (2013); doi: 10.5162/sensor2013/A3.2

[3] H. Lamb, On waves in an elastic plate, Proceedings of the Royal Society of London. Series A, Containing papers of a mathematical and physical character, 114-128 (1917); doi: 10.1098/rspa.1917.0008

[4] D.N. Alleyne, P. Cawley, A 2-dimensional Fourier transform method for the quantitative measurement of Lamb modes, Ultrasonics Symposium IEEE 1990, 1143-1146 (1990); doi: 10.1109/ULTSYM.1990.171541

[5] R. Lerch, G.M. Sessler, D. Wolf, Technische Akustik: Grundlagen und Anwendungen, Springer, (2009); doi: 10.1007/978-3-540-498339

[6] W. Demtröder, Experimentalphysik 1., Springer, (2008); doi: 10.1007/978-3-642-03911-9
[7] E. Ivers-Tiffée, W.v. Münch, Werkstoffe der Elektrotechnik, Vieweg Verlag, (2007); doi: 10.1007/978-3-322-96802-9 